\title{
General Chair
}

Prof. Kingsum Chow, University of Washington USA

\section{Co-Chair}

Prof. Li Zhou, National University of Defense Technology, China

Prof. Junli Zhao, Qingdao University China

\section{Editor}

Prof. Tao Gong, Donghua University, China

Prof. Ting Yang, Tianjin University, China

Prof. Juan Xu, Hefei University of Technology China

\section{Technical Program Committee}

Dr. Imran Memon, College of Computer Science and Technology, Zhejiang University

Dr. Jianzhou Zhao, Cadence Design System, San Jose, United States

Dr. Malka N. Halgamuge, Department of Electrical \& Electronic Engineering, Melbourne School of Engineering, The University of Melbourne, Melbourne, Australia

Dr. Ximing Fu, Department of Computer Science and Technology, Tsinghua University

Dr. NoranAzizan Bin Cholan, Faculty of Electrical and Electronics Engineering, UniversitiTun Hussein Onn Malaysia, BATU PAHAT, Malaysia

Dr. Krzysztof Gdawiec, Institute of Computer Science, University of Silesia, Sosnowiec, Poland China

Dr. Tigang Jiang, University of Electronic, Science and Technology of

Prof. Huamin Wang, Wuhan University, Wuhan, hubei, China

Dr. Ray-Hwa Wong, Department of Mechanical Eng., Hwa-Hsia University of Technology, Taiwan, R.O.C, Taiwan

Dr.Nongqing Yang, Northwest University for Nationalities, China

Dr. Cao Danyang, North industry China University, Beijing, China

Dr.Weilong He, Henan Normal University, China

Dr. Pingping Liu, Beijing Wuzi University Beijing, China

Dr.Xiaodong Wang, East China Jiaotong University, China

Dr. An Bao-Ran, School of Astronautics, Harbin Institute of Technology, Harbin, China

Dr.Yan Shi, Hohai University, China

Dr. Sunwei, College of Information and Control, China

Dr.Xiongkai Shao, Anhui University of Technology, China

Prof. Zhiting Lin, Anhui University, Anhui, China

Dr. Xiang Li, Yunnan University, China

Dr. Tongliang Fan, China Maritime Police Academy, China 
Dr.Yuntao Zhao, Nanjing University of Technology, China

Prof. Xiao-Jun Wu, Department of Computer Science and Technology, China

Dr. Ziyan Guo, Wuhan University, China

Prof. Ming-Chun Tang, Chonqging University Chonqging, China

Dr.Haibo Qian, Hohai University, China

Dr. Qingxiang Fang, China Jiliang University, China

Dr.Jie Zhang, Yangzhou University, China

Prof. Hongzhi Wang, Harbin Institute of Technology, Harbin, China

Dr.Mingfeng Du, Yangzhou University, China

Dr. Lamei Zhang, University:Harbin Institute of Technology, Harbin, China

Dr. Xiuying Li, Heilongjiang University Heilongjiang, China

Dr.Xiaojun Hu, Hangzhou Dianzi University, China

Dr. Kai Wang, Yantai University, Yantai, China

Dr.Aiguo Zhang, South China Normal University, China

Dr. Zhicheng Dong, New campus of Tibet University, Tibet, China

Dr.Yanhong Wu, Qingdao University of Science \& Technology, China

Dr. Yunpeng (Jack) Zhang, Information and Logistics Technology

College of Technology University of Houston, Houston, U.S.

Dr. Gabriel Alungbe, University of Cincinnati

Prof. Jingsong Li, Anhui University, Anhui, China

Dr. Yuetong Lin, Indiana State University

Prof. Hui Yang, Beijing University of Posts and Telecommunications, Beijing, China

Dr. William Grise, Morehead State University

Prof. Yong Xia, School of Computer Science, Northwestern

Polytechnical University (NPU), China

Dr. Jingbo Qiu, AMD Inc, Canada

Prof. Lamei Zhang, University:Harbin Institute of Technology, Harbin, China

Dr. Qing-An Zeng, North Carolina A\&T University

Prof. Xiuying Li, Heilongjiang University Heilongjiang, China

Dr. Tongping Liu, University of massachusetts at Amherst

Dr. Yudi Gondokaryono, Institute of Teknologi Bandung, Indonesia

Dr. Mohd Nazri Ismail, Universiti Kebangsaan, Malaysia

Prof. Xiangtao Li, Northeast Normal University, Jilin, China

Dr. Bensafi Abd-El-Hamid, Abou Bekr Belkaid University of Tlemcen, Algeria

Prof. Anand Nayyar, Punjab Technical University, Jalandhar, India

Dr. Ahmed Nabih Zaki Rashed, Menoufia University, Egypt

Prof. Zhao Wenfeng, Electronics Engineering, South China Agricultural University, China

Dr. Y. R. Ghodasara, Agricultural University, India 
Dr. Shikui Chen, Altair Engineering Inc., California, USA

Dr. Gurjeet Singh, Desh Bhagat Institute of Engineering \& Management, India

Dr. Wang zhendong, jiangxi university of science and technology, China

Dr. Minhthai Tran, Ho Chi Minh City University of Foreign Languages and Information Technology, Ho Chi Minh City, Vietnam

Prof. Xiang Wang, Beijing University of Aeronautics and Astronautics, China

Dr. M V Raghavendra, Adama Science \& Technology University, Ethiopia

Dr. K. Satya Rajesh, Priyadarshi in Institute of Technology \& Management, India

Dr. Xu Wang, Advanced Micro Devices (Shanghai), Co. Ltd. (AMD), China

Prof. Vuda Sreenivasarao, Defence University College, India

Dr. Aziz Bouslikhane, Paul-Verlaine University, France

Dr. Salah Elfaki Elrofai, Sudan University of Science and Technology, Sudan

Prof. Lei Zhang, Chongqing University, China

Dr. FatihEmreBoran, Department of Industrial Engineering, Faculty of Engineering, Gazi University, Ankara, Turkey

Dr. Weimo Liu, Software Engineer/Research Scientist at GraphSQL Inc, China

Dr. R. Panneer Selvam, Indian Institute of Technology Madras, India

Dr. Moon Ho Kim, Chonbuk National University, South Korean

Dr. Alojz Poredo, University of Ljubljana, Slovenia

Prof. Chengyu Liu, Shandong University, China \& Emory University, U.S.

Dr. Sri YulisBinti M Amin, UniversitiTun Hussein Onn Malaysia, BATU PAHAT, Malaysia

Dr. Longsheng Fu, Northwest A\&F University, Yangling, China

Dr. Hui Yang, Beijing University of Posts and Telecommunications, Beijing, China

Dr. Wei Wei, Mathematics and System Sciences, Beijing University of Aeronautics and Astronautics, China

Dr. T. BhuvanEswari, Faculty of Engineering and Technology, Multimedia University, Melaka, Malaysia

Dr. Xiangjie Kong, School of Software, Dalian University of Technology, Dalian, China

Dr. Xiang Chen, Nantong University, Nantong, China

Dr. Kai Tao Nanyang, Technological University, Singapore

Dr. Lainchyr Hwang, Dept. of Electrical Engineering, I-Shou University, Kaohsiung, Taiwan

Dr.Nongqing Yang, Northwest University for Nationalities, China 
Dr. Yilun Shang, Department of Mathematics, Department of Mathematics, Shanghai, China

Dr. Thang Trung Nguyen, Ton Duc Thang University, Ho chi Minh, Vietnam

Dr.Weilong He, Henan Normal University, China

Dr. Chichang Chen, Department of Information Engineering, I-Shou University, Kaohsiung, Taiwan

Dr. Tomasz Andrysiak, Technology and Science University, Bydgoszcz, Poland

Dr.Xiaodong Wang, East China Jiaotong University, China

Dr.Yan Shi, Hohai University, China

Dr. Rayhwa Wong, Department of Mechanical Eng., Hwa-Hsia University of Technology, New Taipei City, Taiwan

Dr. Muhammad Naufal Bin Mansor, KampusUnicitiAlam,Universiti Malaysia Perlis (UniMAP), Sungai Chuchuh, Malaysia

Dr.Xiongkai Shao, Anhui University of Technology, China

Dr. Xiang Li, Yunnan University, China

Dr. Michal Kuciej, Faculty of Mechanical Engineering, Bialystok University of Technology, Bialystok, Poland

Dr. Imran Memon, Zhejiang university, Hangzhou, China

Dr. Yosheng Lin, National Chi Nan University, Nantou, Taiwan

Dr.Yuntao Zhao, Nanjing University of Technology, China

Prof. SerdarEthemHamamci, Electrical-Electronics Engineering Department, Inonu University, Malatya, Turkey

Dr. Fuchien Kao, Da-Yeh University, Zhanghua County, Taiwan

Dr. Ziyan Guo, Wuhan University, China China

Dr. Zhiyu Jiang, University of Chinese Academy of Sciences, Beijing,

Dr. WananXiong, School of Electronic Engineering, University of Electronic Science and Technology of China(UESTC), Chengdu, China

Dr. Dandan Ma, University of Chinese Academy of Sciences, Beijing, China

Dr.Haibo Qian, Hohai University, China

Dr. ChienhungYeh, Department of Photonics, Feng Chia University, Taichung, Taiwan

Dr. Adam Głowacz, AGH University of Science and Technology, Cracow, Poland

Dr.Jie Zhang, Yangzhou University, China

Dr. Osama Ahmed Khan, Lahore University of Management Sciences, Lahore, Pakistan

Dr. Xia Peng, Microsoft, Boston, United States

Dr. Andrzej Glowacz, AGH University of Science and Technology, Cracow, Poland

Dr.Mingfeng Du, Yangzhou University, China 
Dr. Zhuo Liu, Computer Science and Software Engineering Department, Auburn University Auburn, United States

Dr. ZuraidiSaad, Universiti of Teknologi MARA, Shah Alam, Malaysia

Dr. Gopa Sen, Institute for Infocomm Research, Agency for Science

Technology and Research (A*STAR), Singapore

Dr.Lili Niu, Yibin University, China

Dr. Muhammed EnesBayrakdar, Department of Computer Engineering, Duzce University, Duzce, Turkey

Dr. DeepaliVora, Department of Information Technology, Vidyalankar Institute of Technology, Mumbai, India

Dr. Xu Wang, Shanghai Jiao Tong University, Shanghai, China

Dr. Quanyi Liu, School of Aerospace Engineering, Tsinghua University, Beijing, China 